that will test whether an anti-amyloid agent can slow cognitive decline during the preclinical stage of AD.

\section{O1-02-03 DIAN-TU ADAPTIVE PREVENTION TRIAL \\ LAUNCH AND BASELINE DATA}

Randall J. Bateman ${ }^{1,2,3}$, Susan Mills ${ }^{1}$, Anna Santacruz ${ }^{1}$, Martin R. Farlow ${ }^{4}$, Stephen Salloway ${ }^{5}$, John C. Morris ${ }^{3,6,7}$, Tammie L. S. Benzinger ${ }^{1,2}$, Anne M. Fagan ${ }^{6,7}$, Alison Goate $^{8}$, Jason Hassenstab ${ }^{1}$, Guoqiao Wang ${ }^{1}$, Chengjie Xiong ${ }^{1}$, Clifford R. Jack, Jr, ${ }^{9}$, Bob Koeppe ${ }^{10}$, Ferenc Martenyi ${ }^{11}$, Alison Searle ${ }^{11}$, Roy Yaari $^{12}$, Eric Siemers ${ }^{12}$, Eric McDade ${ }^{1,2}$, Table 1

Demographics
David Clifford ${ }^{1},{ }^{1}$ Washington University in St. Louis School of Medicine, St. Louis, MO, USA $;^{2}$ Knight Alzheimer's Disease Research Center, St. Louis, MO, USA; ${ }^{3}$ Hope Center for Neurological Disorders, St. Louis, MO, USA; ${ }^{4}$ Indiana Alzheimer Disease Center, Indianapolis, IN, USA; ${ }^{5}$ Alpert Medical School of Brown University, Providence, RI, USA; ${ }^{6}$ Washington University School of Medicine, Saint Louis, MO, USA, ${ }^{7}$ Knight Alzheimer Disease Research Center, Saint Louis, MO, USA; ${ }^{8}$ Icahn School of Medicine at Mount Sinai, New York, NY, USA; ${ }^{9}$ Mayo Clinic, Rochester, MN, USA; ${ }^{10}$ University of Michigan, Ann Arbor, MI,

\begin{tabular}{|c|c|c|c|c|}
\hline & All Amyloid PET participants & Not Elevated Amyloid (A $\beta-)$ & Elevated Amyloid $(\mathrm{A} \beta+)$ & \\
\hline & $N=4487$ & $N=3164$ & $N=1323$ & P-value ${ }^{\S} \mathrm{A} \beta$-vs $\mathrm{A} \beta+$ \\
\hline Age - Mean years (S.D.) & $71.29(4.67)$ & $70.95(4.53)$ & $72.10(4.89)$ & $<0.0001$ \\
\hline Sex & & & & 0.689 \\
\hline $\mathrm{F}$ & $2656(59 \%)$ & $1879(59 \%)$ & $777(59 \%)$ & \\
\hline Racial Categories & & & & \\
\hline Native Hawaiian/Pacific Islander & $2(0 \%)$ & $2(0 \%)$ & $0(0 \%)$ & $1.000^{1}$ \\
\hline Black/African American & $167(4 \%)$ & $133(4 \%)$ & $34(3 \%)$ & $0.029^{1}$ \\
\hline White & $4116(92 \%)$ & $2866(91 \%)$ & $1250(94 \%)$ & $<0.001^{1}$ \\
\hline Unknown/Not Reported & $26(1 \%)$ & $18(1 \%)$ & $8(1 \%)$ & $1.000^{1}$ \\
\hline Ethnicity & & & & 0.192 \\
\hline Hispanic or Latino & $142(3 \%)$ & $103(3 \%)$ & $39(3 \%)$ & \\
\hline Divorced & $628(14 \%)$ & $438(14 \%)$ & $190(14 \%)$ & \\
\hline Widowed & $426(9 \%)$ & $304(10 \%)$ & $122(9 \%)$ & \\
\hline Never married & $183(4 \%)$ & $135(4 \%)$ & $48(4 \%)$ & \\
\hline Unknown & $83(2 \%)$ & $63(2 \%)$ & $20(2 \%)$ & \\
\hline Participant Retired & $3402(76 \%)$ & $2397(76 \%)$ & $1005(76 \%)$ & 0.927 \\
\hline Family History of Dementia & $3112(69 \%)$ & $2136(68 \%)$ & $976(74 \%)$ & 0.001 \\
\hline APOE Genotype & & & & \\
\hline$\varepsilon 2 / \varepsilon 2$ & $25(1 \%)$ & $23(1 \%)$ & $2(0 \%)$ & $0.030^{1}$ \\
\hline$\varepsilon 2 / \varepsilon 3$ & $448(10 \%)$ & $379(12 \%)$ & $69(5 \%)$ & $<0.001^{1}$ \\
\hline$\varepsilon 2 / \varepsilon 4$ & $116(3 \%)$ & $74(2 \%)$ & $42(3 \%)$ & $0.121^{1}$ \\
\hline$\varepsilon 3 / \varepsilon 3$ & $2411(54 \%)$ & $1930(61 \%)$ & $481(36 \%)$ & $<0.001^{1}$ \\
\hline$\varepsilon 3 / \varepsilon 4$ & $1292(29 \%)$ & $683(22 \%)$ & $609(46 \%)$ & $<0.001^{1}$ \\
\hline
\end{tabular}

${ }^{\S}$ Fisher's Exact test for categorical variables and Two-sample t-test with unequal variances were used for continuous variables.

${ }^{1}$ Comparisons across individual Racial Categories and APOE Genotyope sub-groups were done using a Fisher's Exact test with a Holm's adjustment to the pvalue to account for multiple comparisons.

Table 2

Cognitive Tests and Subjective Report of Functional Decline

\begin{tabular}{|c|c|c|c|c|}
\hline & $\frac{\text { All Amyloid PET participants }}{N=4487}$ & $\frac{\text { Not Elevated Amyloid (A } \beta-)}{N=3164}$ & $\frac{\text { Elevated Amyloid }(\mathrm{A} \beta+)}{N=1323}$ & $\begin{array}{l}\text { P-value A } \beta \text {-vs. A } \beta+ \\
\text { (Covariate Adj*) }\end{array}$ \\
\hline $\begin{array}{l}\text { PACC - Sum of z-scores Mean (Standard } \\
\text { Deviation) }\end{array}$ & $0.00(2.54)$ & $0.18(2.45)$ & $-0.43(2.68)$ & $<0.0001(<0.0001)$ \\
\hline MMSE & $28.81(1.21)$ & $28.84(1.18)$ & $28.73(1.28)$ & 0.0115 (0.0789) \\
\hline FCSRT Free & $28.96(5.59)$ & $29.28(5.49)$ & $28.21(5.75)$ & $<0.0001(<0.0001)$ \\
\hline FCSRT Total & $47.37(0.91)$ & $47.41(0.88)$ & $47.28(0.99)$ & $<0.0001(0.0005)$ \\
\hline CFI-Self & $2.03(2.08)$ & $1.89(2.01)$ & $2.36(2.20)$ & $<0.0001(<0.0001)$ \\
\hline CFI-Study Partner & $1.26(1.85)$ & $1.17(1.77)$ & $1.49(2.02)$ & $<0.0001(<0.0001)$ \\
\hline
\end{tabular}

* Analysis of Covariance on screening scores, adjusted for screening age, gender and years of education. 
USA; ${ }^{11}$ Roche/Genentech Product Development, Neuroscience, Basel, Switzerland; ${ }^{12}$ Lilly Research Laboratories, Indianapolis, IN, USA.Contacte-mail: batemanr@wustl.edu

Background: Alzheimer's disease (AD) prevention trials target an earlier stage of the disease based on the idea that earlier intervention before neuron loss and symptom onset will provide improved outcomes. The Dominantly Inherited Alzheimer Network Trials Unit (DIAN-TU) launched the first AD prevention trial in 2012 in a rare population of dominantly inherited AD mutation carriers who are destined to get the disease with near $100 \%$ penetrance. Recruitment was completed in 2015 into two parallel drug arms in the DIAN-TU adaptive prevention trial (DIAN-TU APT) platform. A major goal of public-private prevention trials is to make data available to the research community and this presentation will provide comprehensive results of baseline data. Methods: The metrics of establishing the DIAN-TU platform, start-up of sites, launch of the trial, screening, enrollment and close of enrollment measures were analyzed. Baseline demographic, clinical, cognitive, genetic, imaging including MRI, amyloid PIB PET, amyloid AV45 PET, tau AV1451 PET and biomarker results were analyzed according to protocol. Measures were compared to prior findings in the DIAN observational study. A process for DIANTU data requests was developed, approved, and activated in 2017. Results: The DIAN-TU APT platform was established in the first year with protocol, site, operational, and multiple partner start-up in this global trial. As sites were activated, enrollment rate increased with rapid enrollment by the end of the study. 194 participants enrolled to successfully meet enrollment goals within projected timelines. Screen fail rate (19\%), recruitment source (47\% DIAN-obs, 38\% DIAN Expanded Registry), and completion rates of all baseline assessments (99-100\%) were excellent. This trial provides comprehensive clinical, cognitive, imaging and biomarker data and samples that are being used in the final analyses and are available to address important scientific and medical questions. Conclusions: Clinical prevention trials in $\mathrm{AD}$ with multiple $\mathrm{AD}$ biomarkers are feasible and can be highly successful, even in a rare population. The results from comprehensive evaluations during trials can provide unique insights into the effects of interventions and promise to accelerate highly effective treatments and preventions for AD.

\section{O1-02-04 BASELINE CHARACTERICS FROM A PHASE 3 TRIAL OF CRENEZUMAB IN PRODROMAL TO MILD ALZHEIMER'S DISEASE (CREAD)}

Helen Lin ${ }^{1}$, Susanne Ostrowitzki ${ }^{1}$, Kaycee M. Sink ${ }^{1}$, Laurie Millar ${ }^{2}$, Francis Warren ${ }^{2}$, Jillian Smith ${ }^{2}$, Andres Schneider ${ }^{3}$, Reina N. Fuji ${ }^{1}$, Angelica Quartino ${ }^{1}$, Howard Mackey ${ }^{1}$, Michael A. Rabbia ${ }^{4}$, Susan Yule ${ }^{3}$, Paulo Fontoura ${ }^{3}$, Rachelle Doody ${ }^{1,5},{ }^{1}$ Genentech, Inc., South San Francisco, CA, USA; ${ }^{2}$ Roche Products Ltd., Welwyn Garden City, United Kingdom; ${ }^{3}$ F. Hoffmann-La Roche Ltd, Basel, Switzerland; ${ }^{4}$ Genentech, Inc., Little Falls, NJ, USA; ${ }^{5}$ Product Development Neuroscience, F. Hoffmann-La Roche Ltd, Basel, Switzerland. Contacte-mail: lin.helen@ gene.com

Background: Crenezumab is a humanized anti-amyloid-beta $(\mathrm{A} \beta)$ monoclonal IgG4 antibody in development for Alzheimer's disease (AD). Crenezumab binds to multiple forms of $\mathrm{A} \beta$, with high affinity for oligomers, blocking oligomer-induced neurotoxicity, and with low risk of amyloid-related imaging abnormalities (ARIA). Although Phase 2 co-primary endpoints were not met, exploratory analyses suggested that crenezumab should be tested for clinically meaningful efficacy at a higher dose and earlier disease stage. Data from a Phase $1 \mathrm{~b}$ study that investigated the safety/tolerability of higher doses of crenezumab supported a 4-fold higher Phase 3 dose than used in Phase 2. Two global, randomized, double-blind, placebo-controlled, parallel-group Phase 3 studies (CREAD [NCT02670083]; CREAD2 [NCT03114657]) are testing the efficacy and safety of crenezumab $(60 \mathrm{mg} / \mathrm{kg})$ in patients with prodromal to mild AD. Here we describe the study design/methodology, and baseline characteristics from CREAD. Methods: Patients aged 50-85 years with prodromal to mild $\mathrm{AD}$ and confirmed evidence of cerebral amyloid pathology (CSF or amyloid PET) were enrolled. At screening, patients had an MMSE score of $\geq 22$, a CDR-global score of 0.5 or 1 , Free and Cued Selective Reminding Test (FCSRT) immediate free recall $\leq 27$ and cueing index $\leq 0.67$ (to enrich for patients with greater likelihood of progression over 105 weeks), and were randomized $1: 1$ to placebo or crenezumab (60 mg/kg q4w IV). Randomization was stratified by dementia and APOE status, baseline anti-dementia medications, and geographic region. Primary and secondary endpoints include change from baseline in CDRSB, ADAS-Cog-13, and ADCS-ADL scores over 105 weeks. Exploratory objectives are to assess treatment effects on CSF biomarkers and amyloid- and tau-PET. MRI examinations are used to monitor safety and measure volumetric changes. Results: The CREAD study has completed recruitment, with 813 patients enrolled. Baseline data will be presented. Conclusions: Building on learnings from Phase 2, the CREAD and CREAD2 Phase 3 trials are investigating the clinical efficacy of a 4-fold higher dose of crenezumab (vs. Phase 2) in prodromal to mild AD, and will test whether clinically meaningful efficacy can be achieved without the associated safety findings that have been described with other passive anti-amyloid immunotherapies targeting fibrillar amyloid in AD.

\section{1-02-05 PREDICTING AMYLOID BURDEN IN SCREENING FOR PRECLINICAL AD PREVENTION TRIALS}

Michael C. Donohue ${ }^{1,2}$, Rema Raman ${ }^{1}$, Chung-Kai Sun ${ }^{2}$, Oliver Langford $^{1,2}$, Keith A. Johnson ${ }^{3}$, Paul S. Aisen ${ }^{2}$, Reisa A. Sperling ${ }^{4},{ }^{1}$ University of Southern California, San Diego, CA, USA ${ }^{2}$ Alzheimer's Therapeutic Research Institute, San Diego, CA, USA $;{ }^{3}$ Department of Radiology, Division of Molecular Imaging and Nuclear Medicine, Massachusetts General Hospital, Boston, MA, USA $;{ }^{4}$ Brigham and Women's Hospital, Harvard Medical School, Boston, MA, USA. Contact e-mail: mdonohue@usc.edu

Background:Current secondary prevention trials are recruiting participants with evidence of elevated amyloid burden using amyloid PET imaging. Here we explore the best combination of predictors of florbetapir PET SUVR using screening data from the Anti-Amyloid Treatment in Asymptomatic AD (A4) Study. The resulting prediction algorithm could be useful as a prescreen to reduce the number of PET scans required in a future secondary prevention trial. Methods: We apply random forest machine learning methods using demographic variables and measures of cognition and function. We consider models with and without APOE genotype. We evaluate the estimated out-of-sample predictive accuracy of each random forest in predicting amyloid burden in independent validation sets while maintaining a detection prevalence of 50\% (proportion predicted to be $\mathrm{A} \beta+$ ). Results: Figure 1 shows ROC curves for 